Cahiers de recherches médiévales

\title{
La figure mythique du cauchemar
}

Une écrasante présence

\section{Bernard Terramorsi}

\section{(2) OpenEdition Journals}

Édition électronique

URL : https://journals.openedition.org/crm/1723

DOI : $10.4000 / \mathrm{crm} .1723$

ISSN : 1955-2424

Éditeur

Honoré Champion

\section{Édition imprimée}

Date de publication : 15 décembre 2004

Pagination : 46-55

ISSN : $1272-9752$

Référence électronique

Bernard Terramorsi, «La figure mythique du cauchemar », Cahiers de recherches médiévales [En ligne], 11 | 2004, mis en ligne le 06 mars 2008, consulté le 15 décembre 2022. URL : http:// journals.openedition.org/crm/1723; DOI : https://doi.org/10.4000/crm.1723

Ce document a été généré automatiquement le 15 décembre 2022.

Tous droits réservés 


\title{
La figure mythique du cauchemar
}

\author{
Une écrasante présence
}

\section{Bernard Terramorsi}

1 Le cauchemar se caractérise chez un dormeur par une sensation d'étouffement et d'impotence associée à une vision onirique terrifiante. Entre la démonologie et la médecine, la notion moderne de cauchemar a peu à peu émergé au cours du XIX ${ }^{\mathrm{e}}$ siècle où on l'identifie finalement comme une crise d'angoisse nocturne provenant d'une passion amoureuse contrariée. On pense alors que les pulsions font retour dans et par le rêve et sont projetées dans l'agression subie d'un démon lubrique qui chevauche le dormeur. Au début du $\mathrm{XX}^{\mathrm{e}}$ siècle, Freud analysera le cauchemar comme un épisode psychotique réversible, constituant pour le sommeil ce que le délire est à la veille.

2 La croyance originelle voulant que le cauchemar ne soit pas un rêve mais un mort malfaisant s'est estompée dans les mentalités modernes; on peut incriminer l'oubli progressif du fonds légendaire médiéval, la fascination exercée par la figure totalisante du vampire ${ }^{1}$, et enfin la vulgarisation des théories freudiennes sur le rêve. Mais la littérature - autrement savante-, a su conserver le personnage mythique du cauchemar, qui hante nombre de textes romantiques et réapparaît encore dans des fictions modernes. C'est ce que notre étude s'attachera principalement à exposer à partir de la lecture d'œuvres littéraires et picturales.

3 Pour les Anciens, le cauchemar - on disait la quauquemaire jusqu'au XV siècle, puis la cauchemare jusqu'au XVII ${ }^{\mathrm{e}}$ siècle -, ne désignait pas simplement un rêve effrayant. Le cauchemar était le nom d'un mort maléfique - souvent associé à un cheval surnaturel qui revenait du monde des morts la nuit et profitait du sommeil de sa victime pour la chevaucher et l'écraser de son corps abject. Dès l'Antiquité, les croyances populaires attribuent le cauchemar à Hécate, même si Hippocrate met en cause les humeurs et préfère penser que la dyspnée et le malaise du dormeur trouvent leur origine dans une indigestion, et préconise en conséquence une hygiène alimentaire adaptée le soir ${ }^{2}$. Pour la médecine antique, le cauchemar est un symptôme qui annonce des maladies graves comme l'épilepsie et pouvant conduire à la mort. Ce rêve traumatisant accompagné de la sensation d'un poids écrasant sur l'épigastre a très tôt frappé les imaginations : un être sur ma poitrine, pesant et impensable ; un corps à corps répugnant, terrifiant avec 
une chose hors représentation mais pourtant là... L'appel au surnaturel opéré par la croyance ou la légende permet de donner du sens à ce qui autrement ne serait qu'une pesée hors de pensée, du pur effroi.

4 Sophie Bridier, reprenant les travaux de Claude Lecouteux, a bien montré que « c'est au Moyen Âge que le cauchemar passe du statut de maladie à celui de phénomène diabolique. Dans l'ensemble des mutations qu'a pu connaître l'histoire du cauchemar, celle-ci est peut-être la plus importante [...]. Puis du domaine de la démonologie, il passe à celui de la psychiatrie à la fin du XIX ${ }^{\mathrm{e}}$ siècle $»^{3}$.

5 Au Moyen Âge en effet, sous la tutelle de l'Église qui christianise en les amalgamant les entités du folklore populaire ou bien les diabolise, le cauchemar prend son caractère satanique. Un scénario stable diffusé par la tradition orale mêlant la mort et l'amour, la terreur et le sexe; et une fascination collective qui s'amplifie avec les siècles font du cauchemar une figure mythique qui imprégnera la littérature et l'iconographie romantiques du XIX ${ }^{e}$ siècle et persistera dans la littérature du $\mathrm{XX}^{\mathrm{e}}$ siècle.

6 Associé par l'Église aux nains et aux elfes en tant que créature de la mort, le cauchemar est diabolisé avec eux aux VII ${ }^{\mathrm{e}}-\mathrm{IX}{ }^{\mathrm{e}}$ siècles; si au XIII ${ }^{\mathrm{e}}$ les nains deviennent des génies domestiques espiègles, le cauchemar inassimilable par le christianisme, est renvoyé à la surnature noire ${ }^{4}$.

7 Les recherches minutieuses de Claude Lecouteux permettent aujourd'hui de distinguer le cauchemar des autres créatures surnaturelles à l'origine des peurs de l'homme médiéval. Au cœur des légendes liées à la crainte immémoriale des morts, le cauchemar se distingue comme un double ou un proche parent qui revient du monde des morts, "se jette sur le dormeur et l'écrase de son poids ${ }^{5}$. Il n'est plus à partir du Moyen Âge " une forme de songe fort désagréable mais une entité qui se jette sur vous la nuit et provoque une sensation d'étranglement [...]; une créature maléfique qui afflige les dormeurs et possède a priori trois dimensions, un poids, des sentiments et une volonté $»^{6}$.

8 Le célèbre tableau d'Heinrich Füssli, The Nightmare - œuvre obsédante dont l'artiste peignit sept versions ${ }^{7}$ à partir du prototype de 1781 -, est à la fois le relais et le catalyseur de cette figure médiévale terrifiante. Au tournant du XVIII ${ }^{\mathrm{e}}$ siècle et du XIX ${ }^{e}$ siècle, cette représentation contemporaine des Caprichos de Goya et de la littérature gothique, matérialise une croyance et fixe toute une oralittérature. La mythologie populaire et savante du cauchemar se trouve ici synthétisée, fixée et réactualisée, à tel point que le tableau conçu comme « un texte pictural ${ }^{8}$ sera cité à l'envi par la littérature fantastique du XIX ${ }^{\mathrm{e}}$ siècle.

9 Claude Lecouteux en étudiant les petits démons de la mythologie populaire médiévale combattus par l'Église, a pu établir au bout d'une longue enquête philologique et historique que

le/la Mahr se situe au croisement de deux traditions, l'une populaire et autochtone, l'autre savante et importée. [...] L'Église reprend et modifie les croyances romaines, les diffuse dans tout l'Occident par le biais des missions et des écrits. [...] Il se forme alors un amalgame dans lequel l'opinion des clercs se superpose à celle des autochtones avant de l'éliminer. [...]Compris comme une maladie, le cauchemar tombe dans le domaine du démon et de la sorcellerie, alors qu'il s'agit d'un être appartenant à la famille des elfes et des nains. [...] Il est probable que les lettrés du Moyen Âge ont connu le sens étymologique du terme incubus... ${ }^{9}$ 
L'auteur poursuit que "le cauchemar est une personnification des mauvais rêves, notamment de ceux qui provoquent une détresse respiratoire [...] à l'origine c'est la matérialisation de la figure d'un mort qui possède les vivants [...] ce que révèle l'analyse étymologique du vocable "cauchemar ». Cauche- est la forme romane du latin calcare, "fouler, écraser ", et mar- est le mot germanique qui a toujours désigné ce phénomène nocturne. Le ou la Mahr [...] a pour habitude, au Moyen Âge, de "chevaucher» les dormeurs ou de les tuer en les piétinant, fait attesté dès le IX ${ }^{\mathrm{e}}$ siècle $»^{10}$. «Le cheval est couramment confondu avec le/la Ma(h)r ; au Moyen Âge, il se disait marh. En allemand moderne, Mähre signifie "la jument" et Mahr "le cauchemar" "11.

11 On peut avancer que les mirabilia du Moyen Âge étant difficilement accessibles, c'est le Nightmare de Füssli qui pour toute la littérature occidentale du $\mathrm{XIX}^{\mathrm{e}}$ et du $\mathrm{XX}^{\mathrm{e}}$ siècle deviendra à la fin du XVIII siècle l'hypotexte pictural de la figure mythique du cauchemar. En tant que Double malfaisant agissant dans les ténèbres pour cocher sa victime endormie, le cauchemar n'a pu être récupéré par l'Église qui au même moment intègre en les amalgamant et les lénifiant les nombreux démons populaires. Le cauchemar, rejeté ainsi dans la surnature noire, n'a pas perdu son pouvoir fascinant au sortir du Moyen Âge et jusqu'à l'époque industrielle.

12 The Nightmare de Füssli ${ }^{12}$ a d'abord pour vocation d'être l'image synthétique du scénario très référentiel de la quauquemaire médiévale. On peut dire en effet que «la composition picturale de Füssli projette l'ambisémie du signe linguistique [mare, démon et mare, cavale] dans son couple fantomatique [...] dans son discours iconographique illustratif ${ }^{13}$. La toile est bien " la transcription picturale fidèle d'un discours culturel, d'un ensemble d'écrits et de dits composant le concept de " cauchemar " ${ }^{14}$, et constitués peu à peu à l'époque médiévale.

13 Le tableau dont le cadre de la représentation se réduit à une alcôve, montre une jeune fille dans une robe immaculée, étendue sur un lit, la tête basculée dans le vide, le visage torturé. Sur son épigastre trône un être surnaturel inclassable évoquant un gnome ou kobold aux yeux médusants. La cassure impossible au niveau des reins de la virgo dormiens expose un buste presque autonome par rapport au reste du corps ; cette sorte de bloc détaché est le siège du démon côcheur. La jeune fille est brisée par la pression du mar.

Le mar chevauche la jeune fille mais n'a pas un regard pour elle - il la monte puis la laisse tomber. C'est cela qui la brise moralement et physiquement et provoque sa chute. En toile de fond, le rideau de lit est fendu par une tête de cheval exophtalmique contemplant la virgo dormiens et son monteur... Le cheval de Füssli est une bête chthonienne aux yeux gorgonéens, aux naseaux dilatés et aux oreilles cornues, qui pénètre dans l'intimité d'une jeune fille par la fente du rideau. La présence du cheval est d'abord illustrative et redondante par rapport au titre du tableau : night-mare, la jument de la nuit. Elle vient aussi souligner la signification sexuelle du corps à corps sur le lit: le mar est un démon à cheval sur sa victime, et cette chevauchée vaut pour un coït. La cavale - qui pénètre par une fente - est le symbole du transport : un transport des sens - la chevauchée du démonet de la jeune fille, rehaussée par la figure de l'étalon en médaillon.

$\mathrm{Au}$ début du XIX $\mathrm{X}^{\mathrm{e}}$ siècle, dans ce qui à notre connaissance est la première thèse de médecine soutenue sur le cauchemar, Louis Dubosquet soutient :

Le caractère principal de cette affection [le cauchemar] consiste dans le sentiment d'une forte pression que le malade attribue à un poids quelconque, et le plus 
souvent à un être vivant placé sur sa poitrine. Les formes les plus communes sous lesquelles ce poids se présente à l'imagination sont celles d'un cheval monstrueux, d'un homme difforme ou d'une vieille femme qui sauterait sur la poitrine du malade et y resterait couchés ou assis. Cette circonstance mérite cependant d'être notée parce qu'elle est presque constante, et que le nom de la maladie en est dérivé dans la plupart des langues. Le cauchemar s'appelle en langue celtique macherick (le petit fouleur), en grec ephialtès (qui bondit) en latin incubus et incubo (qui se couche dessus); en allemand nachtmaar (cheval de nuit) et nachtmannlein (petit homme de nuit); en anglais night-mare (vieille cavale de nuit); en hollandais et en flamand nacht-merrie (vieille cavale de nuit); en polonais mara (la cavale) [...]; le mot cauchemar lui-même vient des mots calca (basse latinité, pour calcatio) et mar, adouci du celtique march (cheval). ${ }^{15}$

En annexe de cette thèse de médecine qui se réduit à l'exposé de l'étymologie et de la croyance populaire, puis à une compilation de témoignages et de conseils diététiques, on trouve une reproduction du Nightmare de Füssli... En ce début du XIX ${ }^{\mathrm{e}}$ siècle, le jeune médecin qui tente de penser le cauchemar ne peut que produire une enquête philologique, accompagnée d'un choix d'aphorismes d'Hippocrate et de témoignages de seconde main traversant les siècles. En guise de conclusion ou de synthèse, il reproduit le tableau de Füssli sans autre forme de commentaire... La thèse de médecine n'est en fait qu'une glose de ce tableau, lui-même illustration scrupuleuse de la légende médiévale.

Parmi les exemples littéraires empruntés au XIX ${ }^{e}$ siècle, nous choisirons deux œuvres : l'une attendue - Le Horla de Maupassant -, l'autre moins : Le mariage de Loti. Ce récit nous permettra de prolonger notre enquête par l'examen du tableau de Paul Gauguin, L'esprit des morts veille.

Dans Le Horla, Maupassant réfère explicitement à la créature mythique et à son œuvre écrasante, avant de faire de son personnage surnaturel une altérité prête à écraser le monde entier :

un cauchemar m'étreint; je sens bien que je suis couché et que je dors...[...] je sens aussi que quelqu'un s'approche de moi, me regarde, me palpe, monte sur mon lit, s'agenouille sur ma poitrine [...]. Moi je me débats, lié par une impuissance atroce [...] j'essaye avec des efforts affreux, en, haletant, de rejeter cet être qui m'écrase et qui m'étouffe, - je ne peux pas! ${ }^{16}$

Mes cauchemars anciens reviennent. Cette nuit j'ai senti quelqu'un accroupi sur moi et qui, sa bouche sur la mienne, buvait ma vie entre mes lèvres. Puis il s'est levé, repu, et moi je me suis réveillé, tellement meurtri, brisé, anéanti, que je ne pouvais plus remuer. ${ }^{17}$

Il est remarquable que pour expliquer cette agression, le héros de Maupassant en vienne à évoquer « une épidémie de folie comparable aux démences contagieuses qui atteignirent l'Europe au Moyen Âge $»^{18}$ et qui sévirait maintenant au Brésil. Une maladie d'un autre temps - celui d'un Moyen Âge associé au chaos: la peste et le choléra, la famine et les guerres - et d'un pays autre : la dimension exotique du Brésil, sous la plume de l'écrivain, permet d'élaborer un équivalent contemporain et fantasmatique du Moyen Âge. La référence au Brésil - associé fantasmatiquement à la chaleur, aux fièvres et à la jungle - permet de passer sous silence le poids de la forêt normande en bordure immédiate de la maison. Le Brésil lointain et d'un autre âge a quelque chose de diabolique : c'est le «beau trois-mâts brésilien » remontant la Seine et passant devant sa maison qui, selon le héros, aurait transporté l'être surnaturel étouffant, le mal en incubation. 

attractive de "la nouvelle Cythère $»^{19}$, du mythe de Tahiti qui mêle exotisme et érotisme. Sur le conseil de Van Gogh, Paul Gauguin lira le roman paru en 1879 et n'aura de cesse de rallier Tahiti pour le mettre scrupuleusement en application. Le mariage de Loti est surtout connu pour ses descriptions complaisantes d'un paradis insulaire réduit à un "pays de fleurs et de belles jeunes femmes ${ }^{20}$, «l'île la plus voluptueuse de la terre $»^{21}$.

21 Le héros - un jeune marin européen - vit là une idylle polynésienne avec une " petite fille passionnée de quinze ans $»^{22}$. Chaque fois qu'il évoque dans son récit de "petites créatures ${ }^{23}$, il s'agit de jeunes vahinés lascives et non de quauquemaires...

Cependant si on lit l'œuvre attentivement, on remarque des références insistantes aux "Toupapahou, des fantômes tatoués qui sont la terreur de tous les Polynésiens " ${ }^{24}$, «les terribles Toupapahous $»^{25}$. Cette peur qui gagne le texte de Loti - les carnets de Gauguin relayeront plus tard cette obsession ${ }^{26}$ - n'est pas fortuite ; derrière les poncifs coloniaux de l'île tropicale vouée aux plaisirs de Vénus, le roman témoigne d'un intérêt soutenu pour le surnaturel polynésien et particulièrement la peur des revenants en pleine nuit : ainsi fait son apparition une île thanatique, au cœur de l'île érotique.

Avec sa "petite amie sauvage " (166), l'officier de marine ne vit pas seulement une passion charnelle transgressive (Rarahu est à la fois adolescente, maorie et analphabète) : Harry Grant alias Loti s'immerge en effet dans la culture et la langue maories et apprend la peur des morts malfaisants. Une remarque de l'héroïne tahitienne mérite en ce sens notre attention : « quand nous aurons eu peur ensemble, je t'enseignerai en ce qui concerne les Toupapahous, des choses très effrayantes que tu ignores...» (97): l'intimité du couple, l'union de deux êtres d'âge et de culture différents, s'origine dans le partage de l'effroi surnaturel, l'affrontement des démons de la nuit. Dans « la nouvelle Cythère », Hécate est à la croisée des chemins dans la forêt dense, loin des lagons lumineux.

Dans la dernière partie du texte et de son séjour tahitien, le héros initié à la peur va faire l'expérience traumatisante d'un corps à corps immonde qui tranche avec ces ébats voluptueux complaisamment rapportés jusque-là. Malgré la célébration de l'exception tahitienne - bout du monde que la société judéo-chrétienne n'a alors pas encore normalisée -; malgré aussi les gloses pseudo-ethnologiques sur les Toupapaous, et l'immersion profonde dans la société traditionnelle polynésienne, Loti le décivilisé réfère spontanément à la croyance occidentale du cauchemar quand le monde lui pèse... La scène passe souvent inaperçue, la lecture étant éblouie par les poncifs du mythe de Tahiti :

Ma pauvre petite amie sauvage! Souvent en m'éveillant la nuit je la revoyais encore [...]. Mais la nuit, quand je me trouvai seul dans le silence et l'obscurité, un rêve sombre s'appesantit sur moi, une vision sinistre qui ne venait ni de la veille ni du sommeil, - un de ces fantômes qui replient leurs ailes de chauves-souris au chevet des malades, ou viennent sur les poitrines haletantes des criminels. ${ }^{27}$

En quelques mots, la scène mythique du cauchemar occidental surgit et assombrit l'image de l'île des plaisirs : les tourments moraux du marin sur le point d'abandonner sa " petite créature », l'énervement de son corps repu de plaisir, le plein et le manque... provoquent l'arrivée du cauchemar en bonne et due forme. Loti se sent écrasé et tourmenté.

Cahiers de recherches médiévales, 11 | 2004 
Dans une lettre adressée à sa femme, Gauguin explique :

Le contexte narratif - le mythe littéraire de Tahiti qui ouvre sur la figure de la belle des îles - est a priori à l'opposé de l'esthétique cauchemardesque : or c'est le scénario mythique du cauchemar qui est pourtant exposé ici, comme si le surnaturel polynésien et le surnaturel européen entraient en concurrence. Une escalade dans l'horreur qui éclipse totalement le paradis tropical au point ici d'en saper le mythe.

7 Il est remarquable qu'à la page suivante, Loti infléchisse ses visions oniriques et les adapte au contexte géo-culturel : le lagon de Bora-Bora se change alors en un décor gothique et Raharu apparaît comme un fantôme avec « un rire figé des Toupapaous " ${ }^{28}$. La vahiné n'est plus un succube comme il l'a spontanément ressenti en dépit du dépaysement, mais un tupapau.

Paul Gauguin dans son tableau L'esprit des morts veille (Manao tupapau, 1892), sous couvert de peindre la croyance polynésienne ${ }^{29}$ au tupapau, reprendra à la fois le tableau matriciel de Füssli et la légende européenne qui lui servait de support. Gauguin qui s'emploie à revivre les aventures de Loti, et qui veut être appelé Oviri (le sauvage), peint dans sa case traditionnelle sa jeune compagne maorie terrorisée par un tupapau: l'œuvre montre en fait une belle-des-îles visitée par le cauchemar ${ }^{30}$.

Malgré sa volonté proclamée d'illustrer la croyance tahitienne au tupapau - alibi ethnologique pour la peinture d'un nu exotique provocateur -, re-présente la légende européenne du cauchemar. En mettant en scène la peur polynésienne des revenants dépourvue jusqu'alors de modèle pictural autochtone -, et en peignant simultanément son fantasme sexuel entêtant - une nymphette tahitienne effrayée et disponible -, l'artiste a réalisé inconsciemment une réécriture du Nightmare de Füssli, une réactualisation de la figure mythique médiévale occidentale.

Je fis un nu de jeune fille. Dans cette position, un rien, et elle est indécente. Cependant je la veux ainsi, les lignes et le mouvement m'intéressent. Alors je lui donne dans la tête un peu d'effroi. Cet effroi il faut le prétexter sinon l'expliquer et cela dans le caractère de la personne, une Maorie. Ce peuple a de tradition une très grande peur de l'esprit des morts. Une jeune fille de chez nous aurait peur d'être surprise dans cette position. (La femme ici point). Il me faut expliquer cet effroi avec le moins possible de moyens littéraires comme autrefois on le faisait. [...] Il y a quelques fleurs dans le fond, mais elles ne doivent pas être réelles, étant imaginatives. Je les fais ressemblant à des étincelles. Pour le Canaque les phosphorescences de la nuit sont de l'esprit des morts et il y croient et en ont peur. Enfin pour terminer je fais le revenant tout simplement, une petite bonne femme; parce que la jeune fille, ne connaissant pas les théâtres de spirites français, ne peut faire autrement que de voir lié à l'esprit du mort le mort lui-même, c'est-à-dire une personne comme elle. Voilà un petit texte qui te rendra savante auprès des critiques ... ${ }^{31}$ [nous soulignons chaque fois]

31 La lettre de Gauguin tend à la fois à gloser sur l'œuvre - rajouter du texte à une peinture-, et à démarquer le tableau des références occidentales et littéraires. Le peintre s'enlise dans le déni ; il sait bien qu'il a peint une quauquemaire - mêlée de références polynésiennes : la vahiné, l'inscription en maori, les couleurs - mais quand même...

Un siècle après l'œuvre exemplaire de Füssli, elle-même grosse du fonds légendaire médiéval, Gauguin intègre et adapte The Nightmare en utilisant ses observations ethnologiques - le culte des morts et la peur des revenants à Tahiti. Tout en affichant son dédain pour « les moyens littéraires d'autrefois », il use dans son commentaire d'un 
lexique cher aux maîtres de la littérature fantastique: "l'effroi ", "la peur ", «le revenant », « l'esprit des morts », « le mort »...

Gauguin rompt avec l'Europe ${ }^{32}$ et régénère sa peinture par une immersion définitive dans la culture polynésienne; mais sa peinture du sexe et de l'effroi malgré un habillage exotique (la "belle des îles", le «tupapau ») s'enracine dans la légende médiévale occidentale du cauchemar.

On prendra comme dernier exemple de l'inscription de la figure mythique médiévale du cauchemar dans la littérature, une nouvelle publiée en 1982 par l'écrivain francoargentin Julio Cortázar. Le récit fantastique intitulé « Pesadillas » (Cauchemars) situe l'action à Buenos Aires durant le putsch militaire de 1976 : alors que l'armée quadrille la ville pour écraser toute révolte, l'héroïne est clouée dans son lit, prisonnière d'un sommeil agité qualifié de "cauchemar» (pesadilla en espagnol) par ses proches. La jeune fille est la métaphore du corps social argentin écrasé alors sous la botte militaire. Le pluriel du titre, pesadillas, annonce bien qu'il n'y a pas un seul cauchemar, celui de Mecha tétanisée sur le lit. Il y a la pesadilla de dedans et celle de dehors...: le poids familial - elle est un poids pour sa famille et sa famille lui pèse -, et le poids de la répression militaire. Pesadilla en espagnol, vient de pesar, "peser ", de peso, " poids »: on se souvient que la caractéristique première du cauchemar dans la croyance, c'est un excédent de poids. Tout vient de là : un poids mort qui coupe le souffle, une pesée qui ne peut être pensée, pesée.

La seule chose identique jour après jour était Mecha, le poids du corps [el peso del cuerpo] de Mecha dans ce lit [...] écrasée là et les écrasant tous [aplastada y aplastando a todos] depuis plusieurs semaines; ils purent voir tous les trois le frémissement se propager le long du corps de Mecha, vif serpent [rápida serpiente] se faufilant du cou jusqu'aux pieds, mouvements des yeux sous les paupières, légère crispation du visage; on aurait dit que Mecha rêvait et que ce rêve était pénible et désespérant, le cauchemar [la pesadilla] qui revenait encore et encore sans qu'elle puisse le chasser [...], Mecha toute entière envahie par cette autre chose qui prolongeait en quelque sorte leur cauchemar à eux, eux incapables de communiquer; Mecha remuait très légèrement les doigts mais ils sentaient que le cauchemar était encore là, qu'il se prolongeait interminablement; Avant même de la distinguer dans la pénombre il sentit la présence du cauchemar, le frémissement des mains, l'habitant secret [la habitante secreta] se faufilait sous la peau. ${ }^{33}$

Cette longue citation qui concentre les passages les plus significatifs de la nouvelle, montre que dans un récit de la fin du $\mathrm{XX}^{\mathrm{e}}$ siècle le cauchemar est encore un être surnaturel qui possède impunément sa victime. Cortázar en évoquant un habitant secret qui serpente dans Mecha fait du cauchemar une force d'occupation, une créature maléfique ; et le corps de la jeune fille devient une sorte de lieu hanté, de maison prise, occupée. Mecha est habitée, hantée par une créature: cette présence en elle s'apparente à un viol, à une torture physique qui fait de ce pseudo sommeil un supplice.

Parallèlement, le démon qui possède Mecha fait d'elle un cauchemar pour sa famille : à tous les sens du terme, elle est un poids pour eux, elle a une présence écrasante tout en étant impotente.

La jeune fille possédée par le mar est bien l'image forte transmise par le scénario mythique médiéval : elle est ici au centre d'un récit moderne qui en jouant de cette référence culturelle établit un lien de complicité avec le lecteur lettré, et aussi donne à l'horreur moderne une profondeur abyssale. 
39 En effet, la nouvelle de Cortázar est à la fois fantastique et engagée : la chose est suffisamment rare pour être remarquée, car le genre fantastique privilégie plus les troubles mentaux que les troubles politiques ${ }^{34}$. Le récit lie de façon indissociable la mythologie fantastique du cauchemar et la mythologie politique du « Pouvoir fort » : il établit une généalogie entre les puissances surnaturelles de jadis et le pouvoir prétorien, entre la Surnature et l'Histoire. L'oppression surnaturelle propre au cauchemar légendaire est délibérément confondue avec l'oppression politique, le terrorisme d'état.

40 Le cauchemar, l'habitant secret qui assujettit Mecha c'est le spectre de l'Histoire. En ce sens l'œuvre de Cortázar s'inscrit bien dans la modernité et ses peurs : particulièrement la peur d'être le jouet de l'Histoire conçue comme une machinerie dont on a perdu les commandes et qui écrase impunément les individus; une sorte de mécanisme diabolique engendrant une terreur aussi forte que les anciennes puissances surnaturelles.

41 Mais parallèlement, la nouvelle qui associe l'oppression de l'incube et l'oppression militaire, rejoint les grandes peurs de l'homme médiéval occidental exposées par Jean Delumeau $^{35}$ : la peur de la guerre, de la famine et des épidémies qui s'en suivent; la peur du pouvoir féodal arbitraire qui peut semer la mort en toute impunité.

On constate finalement que notre enquête sur les apparitions de la figure légendaire du cauchemar dans les œuvres du XIX et du XX $\mathrm{XX}^{\mathrm{e}}$ siècles, montre que le cauchemar est bien une figure mythique : on a identifié et observé un scénario stable d'un forte densité symbolique qui mêle Eros et Thanatos, et exerce une fascination collective qui traverse les siècles ; cette figure mythique forgée par les peurs de l'homme médiéval occidental, a démontré sa capacité de s'enkyster dans des œuvres diverses sans s'y diluer et en leur conférant au contraire une grande profondeur symbolique.

Le mar déjà là sur moi avant d'avoir pu être mis en langage est une chose qui bloque le souffle autant que la pensée. Un excès de réel qui est un excédant de poids. Le cauchemar n'est pas seulement une apparition, il est une chose qui me touche, s'appuie, pèse, puis écrase et enfin tétanise. Les Anciens de fait ont tenté de domestiquer cette horreur, d'alléger le tourment de la victime, en lui attribuant une causalité et un fonctionnement précis dans et par une croyance.

Ce n'est pas un hasard si la figure mythique du cauchemar est un personnage récurrent de la littérature fantastique : le genre de l'irreprésentable et de l'indicible a très tôt accueilli cette créature née des croyances populaires mais qui reste avant tout, ne l'oublions pas, une sensation forte mêlant le désir et l'effroi.

\section{NOTES}

1. Voir les études récentes sur le mythe littéraire du vampire : Jean Marigny, Sang pour sang: le réveil des vampires, Paris, Découvertes/Gallimard, 1993 ; Antoine Faivre et Jean Marigny (éds.), Les Vampires, colloque de Cerisy, Paris, Albin Michel, 1993 ; Claude Lecouteux, Histoires des vampires, Paris, Imago, 1999. 
2. Voir Hippocrate, "Traité des songes ", Euvres médicales, Lyon, Éditions du Fleuve, t. 3, 1954 ; et « De la maladie sacrée », De l'art médical, Paris, Livre de poche, 1994.

3. Sophie Bridier, Le cauchemar. Étude d'une figure mythique, Paris, Presses de la Sorbonne, 2002, p. 91. L'ouvrage est la version condensée d'une thèse de Littératures comparées soutenue à l'université de La Réunion en novembre 2000 sous la direction de B. Terramorsi.

4. « Le fantôme occidental est né à la fin du XIII e siècle »; « "l'image macabre tend à s'imposer à la fin du Moyen Âge dans les images de revenants ", note encore Jean-Claude Schmitt dans Les revenants. Les vivants et les morts dans la société médiévale, Paris, Gallimard, 1994, p. 241 et p. 243.

5. Claude Lecouteux, "Mara-Ephialtes - Incubus. Le cauchemar chez les peuples germaniques », p. 4 et p. 2.

6. Claude Lecouteux, «Le cauchemar dans les croyances populaires européennes ", Le cauchemar. Mythe, folklore, littérature, arts, B. Terramorsi (éd.), Paris, Nathan, 2003 (sous presse).

7. Heinrich Fussli (Zurich, 1741-Putney Hill 1825), The Nightmare (1781), Huile sur toile 101,6 x 127, Musée de Détroit. Le tableau - dit version de Détroit - fut exposé pour la première fois à la Royal Academy au printemps de 1782 ; il fut gravé par Laurede en 1782, par T. Burke en 1783, par Ville Neuve en 1784 et par W. Raddon en 1827.

8. Christian La Cassagnère, "Image picturale et image littéraire dans la nocturne romantique ", Romantisme, 49, vol. XV, 1985, p. 55.

9. Claude Lecouteux, «Mara-Ephialtes-Incubus », Etudes germaniques, janvier-mars 1987, pp. 12-13.

10. Claude Lecouteux et Philippe Marcq, Les esprits et les morts. Croyances médiévales, Paris, Champion, 1990, pp. 20-21.

11. Claude Lecouteux, Fées, sorcières et loups-garous au Moyen Âge, Paris, Imago, 1992, p. 117.

12. Pour une étude systématique du tableau, voir B. Terramorsi, « Portraits du revenant de poids. Un aperçu du cauchemar en peinture ", Le cauchemar. Mythe, folklore, littérature, arts, B. Terramorsi (éd.). Paris, Nathan, 2003 (sous presse).

13. Christian La Cassagnère, «Image picturale et image littéraire dans la nocturne romantique ", Romantisme (49), vol. XV, 1985, pp. 50-51.

14. Ibid., p. 50, 51, 55.

15. Louis Dubosquet, Dissertation sur le cauchemar, thèse de Médecine soutenue à la Faculté de Médecine de Paris, le 22 juin 1815, Paris, Editions Didot Jeune, p. 2.

16. Maupassant, « Le horla » (seconde version), Le Horla et autres contes inquiétants, Paris, Garnier, 1976, p. 424.

17. Ibid., pp. 427-428.

18. Ibid., p. 442.

19. Louis-Antoine de Bougainville, Voyage autour du monde, Paris, Gallimard, 1962, p. 248.

20. Pierre Loti, Le mariage de Loti, Paris, Flammarion, 1991, p. 68.

21. Ibid., p. 168.

22. Ibid., p. 166.

23. Ibid., p. 56.

24. Ibid., p. 74.

25. Ibid., p. 107 et passim.

26. Paul Gauguin, Noa Noa, Paris, Editions Mille et Une Nuits, 1998. Le carnet de voyage qui tient aussi du livre d'esquisses est écrit en 1894 à Paris durant le bref et ultime séjour en Europe de l'artiste.

27. Pierre Loti, Le mariage de Loti, op. cit., pp. 227-228.

28. Ibid., p. 229.

29. Paul Gauguin, "Ancien culte maori », Oviri. Ecrits d'un sauvage, Paris, Gallimard, «Folio/ Essais », 1974, pp. 82-90. « Lettre à Mette », 8 décembre 1892 et Noa Noa (1893-94). 
30. Bernard Terramorsi, «Portraits du revenant de poids. Un aperçu du cauchemar en peinture ", art. cit.

31. Paul Gauguin, Lettre à Mette, Tahiti 8 décembre 1892, Paul Gauguin. Lettres à sa femme et à ses amis,Paris, Grasset, pp. 271-272.

32. «Il faut partir, en Europe tout est pourri », aurait lancé le peintre devant les pavillons des «Colonies» lors de l'exposition universelle de Paris en 1889. Dans une lettre à Odilon Redon en septembre 1890, il confie: "Madagascar est encore trop près du monde civilisé ; je vais aller à Tahiti et j'espère $y$ finir mon existence ».

33. Julio Cortazar, «Cauchemars", Nouvelles complètes, Paris, Gallimard, 1994, pp. 1000-1003. La nouvelle parut pour la première fois en français dans Le Monde Diplomatique d'octobre 1983, puis sera reprise dans le recueil Heures indues, Paris, Gallimard, 1986.

34. Les premiers récits fantastiques étasuniens sont une exception : la Guerre de l'Indépendance et la Révolution sont au centre de nouvelles comme Rip Van Winkle, La Légende du Val Dormant (Washington Irving, 1819) ou Peter Rugg le disparu (1824-27). Voir Trois récits fantastiques américains, B. Terramorsi (éd.), Paris, José Corti, 1996.

35. Voir Jean Delumeau, «Peur et sédition », La peur en Occident, XIV ${ }^{e}$-XVIII ${ }^{e}$ siècles, Paris, Fayard, 1978, pp. 143-196.

\section{AUTEUR}

BERNARD TERRAMORSI

Université de La Réunion 